\title{
ACIDÚRIAS D-2-HIDROXIGLUTÁRICA E L-2-HIDROXIGLUTÁRICA: UMA REVISÃO DA LITERATURA
}

\author{
D-2-HYDROXYGLUTARIC AND L-2-HYDROXYGLUTARIC ACIDURIAS:
} A LITERATURE REVIEW

\author{
Daiane Grigolo Bardemaker Rodrigues ${ }^{1,2}$, Carmen Regla Vargas ${ }^{1,2}$
}

\footnotetext{
Clin Biomed Res. 2016;36(2):80-91

1 Faculdade de Farmácia, Universidade Federal do Rio Grande do Sul (UFRGS). Porto Alegre, RS, Brasil.

2 Serviço de Genética Médica, Hospital de Clínicas de Porto Alegre (HCPA). Porto Alegre, RS, Brasil.
}

Autor correspondente: Carmen Regla Vargas crvargas@hcpa.edu.br Serviço de Genética Médica, Hospital de Clínicas de Porto Alegre Rua Ramiro Barcelos, 2350 90035-903, Porto Alegre, RS, Brasil.

\section{RESUMO}

As acidúrias D-2-hidroxiglutárica (D-2-HGA) e L-2-hidroxiglutárica (L-2-HGA) são raras doenças neurometabólicas que constituem um grupo de erros inatos do metabolismo. Essas doenças são causadas pela deficiência das atividades enzimáticas da D-2-hidroxiglutarato desidrogenase na D-2-HGA do tipo I ou isocitrato desidrogenase na D-2-HGA do tipo II, e da L-2-hidroxiglutarato desidrogenase na L-2-HGA. Os principais achados clínicos nos pacientes caracterizam-se por sintomas neurológicos, como convulsões, coma e atrofia cerebral. Também ocorrem lesões cerebrais nos gânglios da base (D-2-HGA, L-2-HGA) e cerebelo (L-2-HGA). Bioquimicamente, essas acidúrias caracterizam-se por acúmulo em tecidos e elevada excreção urinária dos ácidos D-2-hidroxiglutárico (na D-2-HGA) e L-2-hidroxiglutárico (na L-2-HGA). Ainda, uma terceira variante bioquímica da acidúria, a D,L-2-hidroxiglutárica (D,L-2-HGA), é caracterizada por excreção aumentada de ambos enantiômeros do ácido 2-hidroxiglutárico. Em modelo animal, estudos de toxicidade dos ácidos $\mathrm{D}$ e L-2-hidroxiglutárico mostraram injúria cerebral, mas não foi elucidado o mecanismo exato causador do dano. Além disso, altos níveis dos ácidos D e L-2-hidroxiglutárico foram encontrados em tumores cerebrais. No entanto, a relação entre a acidúria e o câncer ainda precisa ser esclarecida. Tendo em vista a gravidade da doença, este trabalho teve como objetivo fazer uma revisão bibliográfica acerca do tema, enfatizando as consequências do metabolismo, principalmente para o tecido cerebral, bem como apontar possíveis abordagens terapêuticas.

Palavras-chave: ácido D-2-hidroxiglutárico; acidúria D-2-hidroxiglutárica; dano cerebral; ácido L-2-hidroxiglutárico; acidúria L-2-hidroxiglutárica; tumor

\section{ABSTRACT}

The D-2-hydroxyglutaric (D-2-HGA) and L-2-hydroxyglutaric acidurias (L-2-HGA) are rare neurometabolic diseases that form a group of inborn errors of metabolism. They are caused by a deficiency on the enzyme activities of D-2-hydroxyglutarate dehydrogenase in D-2-HGA type I or isocitrate dehydrogenase in D-2-HGA type II, and L-2-hydroxyglutarate dehydrogenase in L-2-HGA. The main clinical findings in affected patients are related to neurological symptoms, such as convulsions, coma and brain atrophy. Brain injuries also occur in the basal ganglia (D-2-HGA, L-2-HGA) and cerebellum (L-2-HGA). These acidurias are biochemically characterized by the accumulation in tissues and increased urinary excretion of D-2-hydroxyglutaric acid (in D-2-HGA) and L-2-hydroxyglutaric acid (in L-2-HGA). Still, a third biochemical variant of aciduria, called D,L-2-hydroxyglutaric (D,L-2-HGA), is characterized by increased excretion of both enantiomers of 2-hydroxyglutaric acid. In an animal model, toxicity studies on D- and L-2-hydroxyglutaric acids showed brain injury, but the exact mechanism of brain damage was not elucidated. Furthermore, high levels of D- and L-2-hydroxyglutaric acids were found in brain tumors. However, the relationship between cancer and aciduria still needs to be clarified. In view of the severity of the disease, this study aimed to do a literature review on the topic, emphasizing metabolic consequences, particularly for the brain tissue, as well as to identify possible therapeutic approaches.

Keywords: D-2-hydroxyglutaric acid; D-2-hydroxyglutaric aciduria; brain damage; L-2-hydroxyglutaric acid; L-2-hydroxyglutaric aciduria; tumor 
As acidemias ou acidúrias orgânicas constituem um grupo de erros inatos do metabolismo (EIMs) caracterizados pelo acúmulo de um ou mais ácidos orgânicos nos líquidos biológicos e tecidos dos pacientes devido à deficiência da atividade de enzimas do metabolismo de aminoácidos, lipídios ou carboidratos ${ }^{1}$.

Em relação à clínica, os indivíduos afetados por acidúrias orgânicas apresentam, predominantemente, disfunção neurológica em suas mais diversas formas de expressão: regressão neurológica, convulsões, coma, ataxia, hipotonia, hipertonia, irritabilidade, tremores, movimentos coreoatetóticos, tetraparesia espástica, atraso no desenvolvimento psicomotor, retardo mental e outras manifestações. As mais frequentes manifestações laboratoriais são cetonemia, cetonúria, neutropenia, trombocitopenia, acidose metabólica, baixos níveis de bicarbonato, hiperamonemia, hipo e hiperglicemia, acidose lática, aumento dos níveis séricos de ácidos graxos livres e outros ${ }^{2}$. Através do uso de tomografia computadorizada, na maioria dos pacientes foram encontradas alterações de substância branca (hipomielização e/ou desmielização), atrofia cerebral generalizada ou dos gânglios da base (necrose ou calcificação), megaencefalia, atrofia frontotemporal e atrofia cerebelar ${ }^{3}$.

Os ácidos enantioméricos D e L-2-hidroxiglutárico foram primeiramente identificados como constituintes normais da urina humana. Três anos depois, dois novos EIMs foram simultaneamente relatados no Journal of Inherited Metabolic Disease. Chalmers et al. ${ }^{4}$ identificaram um paciente com acidúria D-2-hidroxiglutárica (D-2-HGA), enquanto Duran et al. ${ }^{5}$ descreveram um caso de acidúria L-2-hidroxiglutárica (L-2-HGA). A terceira variante bioquímica da acidúria 2-hidroxiglutárica foi descrita por Montau et al. ${ }^{6}$, que relataram três pacientes com elevada excreção urinária de ácido $D$ e L-2-hidroxiglutárico, denotada pelos autores como acidúria $\mathrm{D}, \mathrm{L}-2$-hidroxiglutárica (D,L-2-HGA). $\mathrm{O}$ ácido L-2-hidroxiglutárico (L-2-HG) ou o seu enantiômero, o ácido D-2-hidroxiglutárico (D-2-HG), apresentam concentrações muito elevadas nos tecidos e líquidos biológicos dos pacientes com L-2-HGA e D-2-HGA, respectivamente. Essas doenças metabólicas são geneticamente distintas e, em geral, transmitidas de forma autossômica recessiva ${ }^{7}$. A D-2-HGA tem aproximadamente 80 casos e a L-2-HGA tem por volta de 300 casos relatados no mundo. Essas acidúrias orgânicas, apesar de raras, são bastante graves ${ }^{8,9}$.

Tendo em vista a gravidade da doença devido à produção de metabólitos tóxicos nos indivíduos afetados, bem como a necessidade de maiores estudos a fim de elucidar os mecanismos fisiopatológicos da disfunção cerebral encontrada nesses pacientes, o presente trabalho teve como objetivo fazer uma revisão bibliográfica acerca do tema, enfatizando as consequências do metabolismo tóxico, principalmente para o tecido cerebral. Além disso, buscou-se apontar possíveis alvos terapêuticos e abordagens terapêuticas utilizadas atualmente. Dessa forma, para realizar a pesquisa foram utilizados artigos publicados nos bancos de dados PubMed e MEDLINE, no período de 2000 a 2015, utilizando-se as palavras-chave: D-2-hydroxyglutaric aciduria, L-2-hydroxyglutaric aciduria and 2-hydroxyglutaric aciduria mechanism.

\section{ACIDÚRIA D-2-HIDROXIGLUTÁRICA}

A D-2-HGA é uma rara doença neurometabólica hereditária caracterizada bioquimicamente pelo acúmulo de D-2-HG nos tecidos e elevada excreção urinária $(1565 \pm 847 \mathrm{mmol} / \mathrm{mol}$ creatinina, controles $=6,0 \pm 3,6 \mathrm{mmol} / \mathrm{mol}$ creatinina) e níveis consideráveis de $\mathrm{D}-2-\mathrm{HG}$ no plasma $(61 \pm 14 \mu \mathrm{mol} / \mathrm{L}$, controles $=0,7 \pm 0,2 \mu \mathrm{mol} / \mathrm{L}$ ) e fluido cerebroespinhal $(15-25 \mu \mathrm{mol} / \mathrm{L} \text {, controles }=0,1 \pm 0,1 \mu \mathrm{mol} / \mathrm{L})^{7,10,11}$. AD-2-HGA apresenta-se sob duas formas distintas: uma com apresentação mais lenta e sintomas mais brandos (D-2-HGA do tipo I) e outra com fenótipo mais severo (D-2-HGA do tipo II). A D-2-HGA do tipo I é causada por mutações no gene DHGDH (MIM\# 600721), que codifica a enzima mitocondrial D-2-hidroxiglutarato desidrogenase (D-2-HGDH). A D-2-HGA do tipo II é causada por mutações de ganho de função no gene IDH2 (MIM\# 613657), que é responsável pela codificação da enzima isocitrato desidrogenase $2\left(\right.$ IDH2) ${ }^{12,13}$. Mutações em D-2-HGDH são autossômicas recessivas, enquanto que na IDH2 são autossômicas dominantes e ocorrem mutações de novo em células germinativas ${ }^{14}$.

Estudos realizados em cultura celular de linfoblastos mostraram que o 2-cetoglutarato (2-KG), um intermediário do ciclo de Krebs/ciclo dos ácidos tricarboxílicos (TCA), é convertido em D-2-HG através da enzima transidrogenase hidroxiácido-oxoácido (HOT), enquanto o D-2-HG é convertido em 2-KG através da enzima D-2-HGDH (Figura 1). Ainda, a HOT catalisa a conversão do gama-hidroxibutirato em semialdeído succínico ${ }^{7}$. No que se refere à D-2-HGA tipo II, a enzima IDH2, responsável pela conversão de isocitrato em 2-KG, possui atividade alterada. Essa enzima mutante tem um ganho de função, pois adquire a capacidade de converter 2-KG em D-2-HG (Figura 1). Dessa forma, pressupõe-se que a atividade da D-2HGDH seja insuficiente para metabolizar o excesso de D-2-HG formado, o que resulta em seu acúmulo 7,15 .

Clinicamente, os pacientes apresentam epilepsia, hipotonia e retardo psicomotor como característica primária. Os grupos são heterogêneos para os 


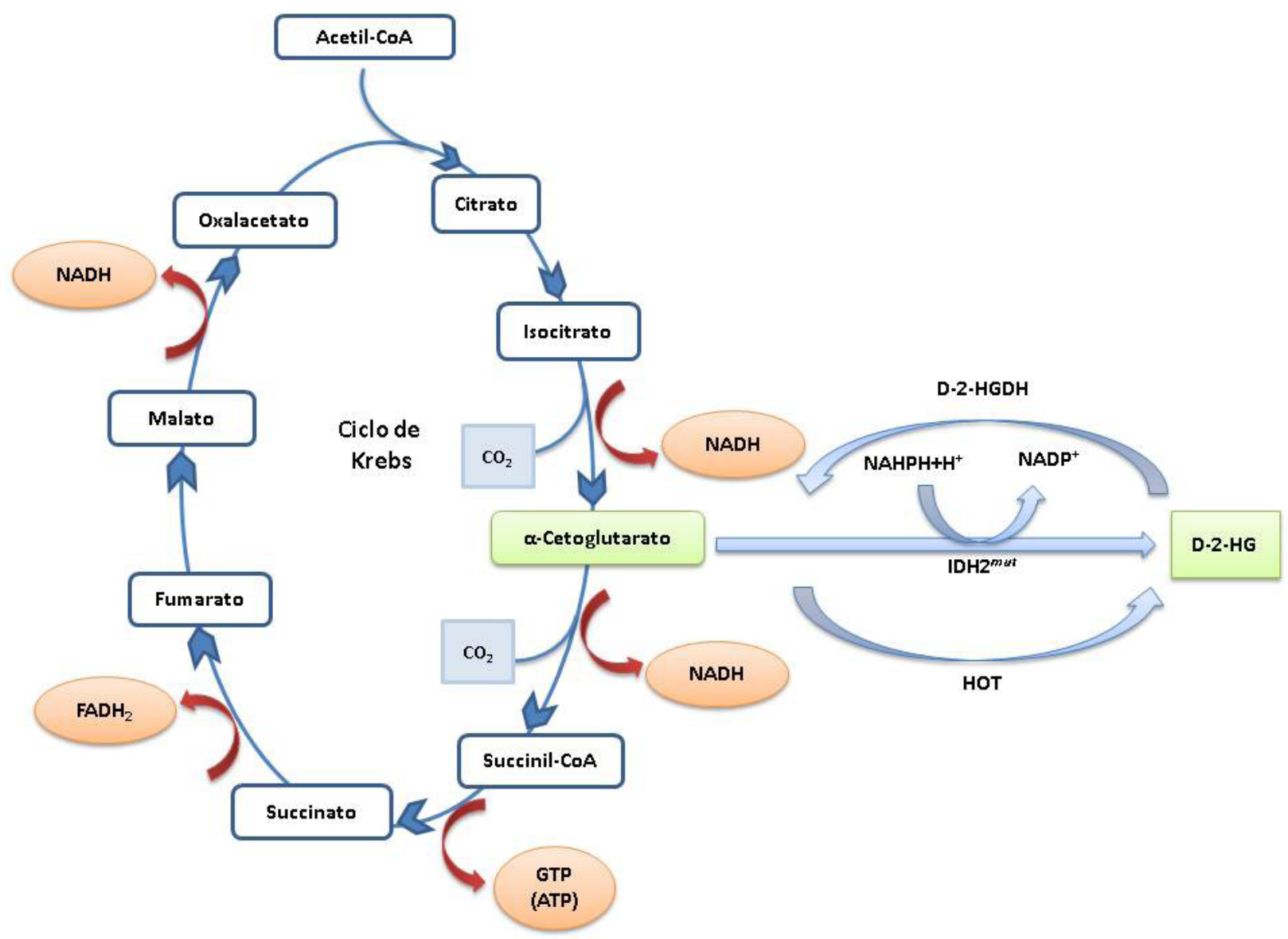

Figura 1: O ácido D-2-hidroxiglutárico (D-2-HG) é formado a partir do a- cetoglutarato (2-KG) via enzima transidrogenase hidroxiácido-oxoácido (HOT). A Enzima D-2-hidroxiglutarato desidrogenase (D-2-HGDH) catalisa a conversão D-2-HG para 2-KG (Na D-2-HGA tipo I ocorre deficiência na D-2-HGDH). A enzima isocitrato desidrogenase 2 mutante (IDH2 ${ }^{M U T}$ ) com ganho de função produz D-2-HG a partir de 2-KG adicionalmente à produção de D-2-HG via HOT. Existe a hipótese de que a D-2-HGDH não é capaz de metabolizar todo o D-2-HG gerado, resultando no acúmulo de D-2-HG (D-2-HGA do tipo II).

subtipos de pacientes, caracterizando a descrição heterogênea de fenótipos brandos ou mais severos. A idade de início dos sintomas para pacientes do tipo I geralmente ocorre nos seis primeiros anos de vida, enquanto que para os pacientes do tipo II os sintomas iniciam por volta dos dois anos de vida. Manifestações comuns para desordens do tipo I e II incluem atraso no desenvolvimento, hipotonia e convulsões, sendo que para o tipo II essas convulsões são mais frequentes, além de o atraso no desenvolvimento ser maior. Ainda, foi observada cardiomiopatia primariamente dilatada em aproximadamente $50 \%$ dos pacientes do tipo II, mas em um dos casos era hipertrófica. Além disso, todos pacientes apresentam, sem diferenciar tipo I ou tipo II, alargamento de ventrículos laterais, alargamento de espaço subaracnóideo frontal, efusão subdural, pseudocistos subependimais, sinais de atraso de maturação cerebral e anormalidades de massa branca cerebral multifocal. Quanto à expectativa de vida, pacientes do tipo II vivem de meses ao início da idade adulta, enquanto que para pacientes do tipo I permanece indefinida ${ }^{7}$.

\section{Toxicidade do ácido D-2-hidroxiglutárico}

Recentes estudos demonstraram que a excitotoxicidade contribui para a neuropatologia da D-2-HGA. Um trabalho realizado em cultura neuronal de células de pintos e ratos mostrou que o D-2-HG, que é estruturalmente similar ao aminoácido excitatório glutamato, induziu dano celular excitotóxico envolvendo a ativação do receptor N-metil-D-aspartato (NMDA). Além disso, para avaliar a ativação de subtipos específicos de receptores NMDA por D-2-HG, foram expressos receptores NR1/NR2A e NR1/NR2B em 
células HEK293, e o ácido foi capaz de ativar esses receptores. Ainda, o D-2-HG inibiu a atividade do complexo $\mathrm{V}$ (ATP sintase) da cadeia respiratória mitocondrial, refletindo um metabolismo energético prejudicado devido à inibição da síntese de ATP; no entanto, o ácido não afetou a atividade catalítica do complexo de transferência de elétrons I-IV ${ }^{16}$.

Estudos em modelos animais vêm sendo realizados na tentativa de elucidar os mecanismos patofisiológicos da doença. Silva et al. ${ }^{17}$ avaliaram o papel do D-2-HG in vitro na atividade da creatina quinase total (CK) de músculo cardíaco e esquelético em ratos Wistar. Foi observado que o ácido inibiu a atividade CK, creatina quinase mitocondrial (mi-CK) e citosólica (ci-CK) do músculo cardíaco e esquelético, sugerindo assim que a inibição dessa enzima por aumento dos níveis do D-2-HG poderia ser um importante mecanismo envolvido na miopatia e cardiomiopatia de pacientes com D-2-HGA. Corroborando esse estudo, da Silva et al. ${ }^{18}$ também avaliaram a atividade da creatina quinase em córtex cerebral de ratos frente ao D-2-HG in vitro. Foi encontrada inibição da atividade da CK pelo ácido, sendo que a fração mitocondrial foi a maior afetada, o que sugere que a inibição dessa enzima por aumento dos níveis do D-2-HG poderia estar relacionada à neurodegeneração encontrada nos pacientes.

Foi demonstrado que o metabolismo energético é marcadamente prejudicado pelo acúmulo do D-2-HG. Latini et al. (2005) avaliaram o papel do ácido na atividade do complexo da cadeia respiratória mitocondrial, além da produção de $\mathrm{CO}_{2}$ no músculo cardíaco e esquelético de ratos Wistar. Os autores observaram a inibição significativa da atividade da ATP sintase e citocromo c oxidase (COX) pelo D-2-HG. Além disso, houve marcada redução da produção de $\mathrm{CO}_{2}$ no músculo cardíaco e esquelético e diminuição significativa, em preparações mitocondriais de cérebro, da taxa de controle respiratório na presença de glutamato/malato e succinato. Também houve redução da viabilidade celular em partes do córtex cerebral. Dessa forma, sugere-se que o metabolismo energético prejudicado poderia contribuir para o entendimento das características clínicas da doença ${ }^{19}$.

Nesse sentido, da Rosa et al ${ }^{20}$ verificaram os efeitos cerebrais do ácido D-2-HG, que foi administrado via intraestriatal de modo agudo in vivo em ratos jovens. Os principais achados da injúria cerebral foram observados após $12 \mathrm{~h}$ e $48 \mathrm{~h}$ da injeção, entre eles vacuolização e infiltração linfocítica e macrofágica. Os autores não elucidaram o mecanismo exato que provocou esse dano, mas hipotetizam que a formação de vacúolo seja secundária a alterações do metabolismo energético, excitotoxicidade e homeostase redox. Dessa forma, a presença de macrófagos e infiltrado linfocítico poderia estar associada ao estresse oxidativo, uma vez que leucócitos são recrutados em resposta à neuroinflamação.

\section{Ácido D-2-hidroxiglutárico e câncer}

Elevados níveis de D-2-HG têm sido detectados em gliomas malignos e leucemia mieloide aguda (LMA) associados a mutações somáticas em genes que codificam isoformas de isocitrato desidrogenase dependentes de NADP $(+)$ citoplasmáticas e mitocondriais, IDH1 e IDH2, respectivamente. Essas mutações ocorrem no códon 132 para IDH1 ou códon 172 e 140 para IDH2, produzindo enzimas deficientes, pois reduzem a afinidade pelo isocitrato e aumentam a afinidade por NADPH e $\alpha-K G$. Isso previne a descarboxilação oxidativa de isocitrato a $\alpha-K G$ e facilita a conversão de 2-KG em 2-HG, consequentemente resultando em altos níveis de D-2-HG nas células ${ }^{21,22}$. Além disso, todas as mutações relatadas em LMA e gliomas resultam na substituição de um resíduo de arginina altamente conservado no sítio ativo da enzima com um diferente aminoácido, ou seja, na proteína IDH1, a mais comum alteração é a substituição do resíduo de arginina pela histidina na posição 132. Correspondente sítio em IDH2, R172, é da mesma forma, frequentemente mutado em gliomas e LMA. Ainda, recentes estudos revelaram que outra mutação em IDH2 resulta da substituição de glutamina por arginina localizada na posição $140^{14}$.

Recente estudo foi realizado utilizando sequenciamento genético do gene IDH2 em um conjunto de amostras de DNA de pacientes com LMA. Como resultado, foram encontrados diversos casos com mutação R172K na IDH2. Ainda, para confirmar se essas células com mutação IDH2 R172K exibem a propriedade de aumentar os níveis de 2-HG, foram utilizadas células expressando IDH2 wild-type ou IDH2 R172K. A determinação foi realizada por cromatografia gasosa acoplada a espectrômetro de massas (CG-MS), e verificou-se um aumento de aproximadamente 100 vezes no nível intracelular de 2-HG em células IDH2 R172k quando comparadas com as células que expressavam o IDH2 wild-type. Além disso, foi confirmada a quiralidade do 2-HG encontrado nas células expressando IDH2 R172K como sendo exclusivamente o enantiômero (R). Além disso, foi evidenciado que o D-2-HG age como mutagênico, pois os dados apresentados nesse estudo, em amostras típicas de LMA com mutações em IDH1 e IDH2, não apresentaram outras mutações conhecidas associadas ao tumor. Ainda, a possibilidade de o 2-HG comportar-se como oncometabólito e, dessa forma, contribuir com a tumorigênese surge do conhecimento de subtipos de tumores em que mutações IDH são encontradas 
com alta frequência. Entretanto, se o 2-HG pertence ao grupo de mutagênicos ou se apenas desempenha alguma função na carcinogênese é um aspecto que precisa ser elucidado. Por outro lado, verificou-se a importância de identificar essas mutações em LMA para melhor entender essa patogênese ${ }^{23}$.

Corroborando essa mesma linha de pesquisa, Gross et al. ${ }^{24}$ verificaram que mutações em IDH1 R132 causam acúmulo de 2-HG em células LMA. Para investigar o papel das mutações IDH1 R132, foi feito sequenciamento genotípico em células de 145 pacientes com LMA. O espectro das mutações difere dos observados no SNC, em que $80-90 \%$ das mutações são IDH1 R132H. No sequenciamento, foram observados cinco pacientes com mutação IDH1 R132H, quatro pacientes com mutação IDH1 132C e dois pacientes com mutação IDH1 R132G. Ainda, um dos pacientes portando mutação IDH1 teve progressão precoce para síndrome mielodisplásica (SMD). Não obstante, foram genotipadas células de pacientes SMD e foram encontradas mutações IDH1 wild-type. Entretanto, é necessária uma quantidade maior de estudos para avaliar se mutações IDH1 contribuem para a progressão da LMA ou se são características desta doença heterogênea.

Os níveis de 2-HG, medidos por cromatografia líquida acoplada a espectrômetro de massas (LC-MS), estavam 50 vezes maiores nas amostras de LMA com mutação IDH1 R132. Além disso, não houve relação entre o aminoácido específico substituído no resíduo 132 e os níveis de 2-HG. Devido ao aumento do 2-HG, foi também sequenciado o gene IDH2 nas amostras, e duas delas apresentaram mutação IDH2 172K. Adicionalmente, foi realizada cultura celular in vitro de células de pacientes com LMA com mutação IDH1 e células wild-type. Com isso, foi observado que os grupos celulares tiveram alta variabilidade e capacidade de proliferar na cultura. Ainda, não houve relação entre os níveis de $2-\mathrm{HG}$ em células mutantes IDH1 R132 na taxa de crescimento e variabilidade na cultura. Após 14 dias em cultura, continuaram a acumular $2-\mathrm{HG}^{24}$.

Além de mutações IDH1 e IDH2 terem sido identificadas em LMA, elas também foram identificadas em gliomas. Muitos estudos observaram mutação em IDH1, R132 ou em seu resíduo análogo R172, em aproximadamente $80 \%$ dos gliomas de baixo grau, anaplásico e glioblastoma secundário. As mutações IDH2 foram menos comuns. Ainda, a maioria das mutações em gliomas são IDH1 R132. Com a modificação da atividade catalítica da enzima, ocorre redução dos níveis de a-KG e NADPH, que são necessários na manutenção da glutationa reduzida (GSH) e prevenção do estresse oxidativo ${ }^{24}$.
Embora tenha sido mostrado que enzimas mutantes IDH1 e IDH2 aumentam os níveis de 2-HG e estão presentes em tumores como LMA e gliomas, ainda é bastante obscuro como essas mutações contribuem para a transformação oncogênica. No entanto, esses achados levam à hipótese de o 2-HG ser um "oncometabólito"25-27.

\section{Ácido D-2-hidroxiglutárico e o envolvimento no estresse oxidativo}

A fisiopatologia da D-2-HGA ainda é pouco conhecida, principalmente em relação ao envolvimento do dano neurológico. No entanto, estudos vêm sendo realizados na tentativa de elucidar o papel das alterações da homeostase redox frente ao acúmulo do ácido.

Um estudo realizado em cultura neuronal de células de pinto investigou o efeito do D-2-HG na concentração de $\mathrm{Ca}^{2+}$ por microscopia de fluorescência usando fura-2, que revelou que o ácido provoca distúrbios na homeostase do $\mathrm{Ca}^{2+}$ intracelular. Além disso, os autores mostraram que esse distúrbio na homeostase do cálcio resulta em aumento da produção de ERO mitocondrial, através do corante sensível a oxidação diidrorodamina-12316.

Recente estudo realizado por da Rosa et al. ${ }^{20}$ demonstrou que o ácido D-2-HG, através de administração intraestriatal aguda, foi capaz de alterar parâmetros de estresse oxidativo em ratos jovens. Nesse estudo, foram administrados in vivo $2,5 \mu \mathrm{mol}$ do ácido e foram observados os seguintes resultados:

a) aumento significativo dos níveis de malondialdeído (MDA), demostrando assim dano oxidativo a lipídios. Sabe-se que o MDA é um produto final da oxidação de ácidos graxos insaturados. Dessa forma, esse aumento pode indicar uma alteração na fluidez e permeabilidade da membrana lipídica das células, o que pode acarretar em alteração de transporte de íons e de processos metabólicos ${ }^{28}$;

b) aumento do conteúdo de carbonilas, mostrando indução de dano proteico. No entanto, esse resultado pode estar associado à perda de função proteica e formação de agregados, que ocorrem quando proteínas sofrem dano oxidativo através da oxidação de cadeias laterais de resíduos de aminoácidos ${ }^{28}$;

c) aumento significativo da produção de nitratos e nitritos, não tendo alterado a oxidação de 2,7-diclorofluoresceína (DCFH) e a produção de peróxido de hidrogênio $\left(\mathrm{H}_{2} \mathrm{O}_{2}\right)$. Dessa forma, conclui-se que são espécies reativas do nitrogênio (ERN) que estão provavelmente envolvidas nos 
efeitos pró-oxidantes, considerando que a DCFH detecta predominantemente espécies reativas do oxigênio $(E R O)^{28}$;

d) redução significativa da concentração de GSH e atividade enzimática de glutationa peroxidase (GPx) e superóxido dismutase (SOD), mostrando assim um comprometimento das defesas antioxidantes cerebrais. Levando-se em conta as funções da GSH como sequestradora de radicais livres e protetora de grupos sulfidrilas, sua concentração é utilizada para avaliar a capacidade tecidual de prevenir o dano oxidativo associado ao processo de produção de radicais livres. Além disso, as atividades enzimáticas da SOD e GPx diminuídas podem ser explicadas pelo ataque oxidativo de espécies reativas em sítios específicos de aminoácidos nas enzimas, causando modificações estruturais que diminuem suas atividades. Cabe salientar que essas alterações na homeostase redox causadas pelo D-2-HGA foram seletivas, tendo em vista que foi utilizado um análogo estruturalmente similar, o ácido 3- metil-glutacônico (3-MGT), o qual não alterou nenhum desses parâmetros ${ }^{20,28}$.

\section{Enzimas IDH1 e IDH2 como alvos terapêuticos}

De acordo com um trabalho realizado em células MCF7, obtidas da American Type Culture Collection (ATCC $^{\circledR}$ ), por Matsunaga et al. (2012), foram mimetizadas células knockdown de IDH1 ou IDH2 em células deficientes D-2-HGDH, mostrando que ocorre diminuição dos níveis do 2HG. No entanto, quando houve expressão de IDH1 ou IDH2 houve um aumento de $2 \mathrm{HG}$, sendo este muito alto em células depletadas de D-2-HGDH. Esses resultados sugerem que IDH1 e IDH2 são responsáveis por produzir D-2HG em condições de diminuição da atividade da D-2-HGDH. Além disso, células knockdown de IDH1 e IDH2 com D-2-HGDH diminuíram os níveis de 2-HG precocemente a níveis residuais, corroborando a hipótese de que essas duas proteínas são as principais fontes de D-2HG em células com supressão da regulação de D-2-HGDH. Neste trabalho, não foi possível analisar o papel da enzima HOT, porque seu mecanismo exato ainda não está totalmente elucidado. Entretanto, este estudo lança uma nova abordagem em relação ao mecanismo da D-2-HGA, permitindo assim indicar possíveis novos alvos terapêuticos, como as enzimas IDH1 e IDH2 como medicações futuras para a D-2-HGA tipo $\mathrm{I}^{29}$. Nessa mesma linha, um estudo realizado em modelo de linfoblastos para atividade de ganho de função da IDH2 apontou essa enzima como possível alvo terapêutico. Mutações do resíduo de arginina para glicina (IDH2 ${ }^{\text {wtR140G }}$ ) ou glutamina (IDH2 ${ }^{\text {wtR140Q }}$ ) têm sido definidas como lesões genéticas primárias em metade dos pacientes com D-2-HGA tipo II. No ensaio, 11 inibidores potenciais (metabólitos endógenos) da atividade enzimática foram avaliados: D-2-HG, L-2-HG, L-isocitrato, citrato, L-glutamato, L-glutamina, L-malato, D-malato, oxaloacetato, acetoacetato e ácido metil-éster-acetoacético. Nesse ensaio, o oxaloacetato foi o mais potente inibidor, e a produção de D-2-HG teve mais de $50 \%$ de redução quando a concentração de inibidor e do substrato foi igual $(15 \mathrm{mM})$. O oxaloacetato agiu como um inibidor competitivo para a conversão de 2-KG em D-2-HG. A afinidade comparável de IDH2 ${ }^{\text {wt/R } 140 Q}$ para oxaloacetato se deve provavelmente à similaridade estrutural ${ }^{15}$.

\section{ACIDÚRIA L-2-HIDROXIGLUTÁRICA}

A L-2-HGA é uma doença neurometabólica que pertence ao grupo das acidúrias orgânicas, com um curso lento e progressivo. Os indivíduos acometidos por essa doença apresentam elevados níveis de L-2-HG em urina $(1283 \pm 676 \mathrm{mmol} / \mathrm{mol}$ creatinina, controles $=6,0 \pm 5,4 \mathrm{mmol} / \mathrm{mol}$ creatinina) e em outros fluidos biológicos, tais como plasma $(47 \pm 13 \mu \mathrm{mol} / \mathrm{L}$, controles $=0,6 \pm 0,2 \mu \mathrm{mol} / \mathrm{L}) \mathrm{e}$ fluido cerebroespinhal $(62 \pm 30 \mu \mathrm{mol} / \mathrm{L}$, controles $=0,7 \pm 0,6 \mu \mathrm{mol} / \mathrm{L})$. A doença tem manifestação durante a infância, mas pode se apresentar na idade adulta com fenótipos mais brandos ${ }^{7,30,31}$. O bloqueio metabólico presente nesses pacientes deve-se à ausência da atividade da enzima mitocondrial L-2-hidroxiglutarato desidrogenase ligada ao FAD (L-2-HGDH), devido a mutações no gene L2HGDH, localizado no cromossomo $14 q 22.1^{32,33}$.

Clinicamente, a L-2-HGA é caracterizada por hipotonia, ataxia progressiva, deficiência mental com graus variados de leucoencefalopatia subcortical e atrofia cerebelar ${ }^{34}$. Ainda, mudanças histopatológicas incluem desmielinização difusa, espongiose e cavitação cística de massa branca cerebral, globo pálido e núcleo denteado. Entretanto, os mecanismos envolvidos em todas essas alterações ainda permanecem obscuros $^{35}$. A L-2-HGA é peculiar, ao contrário de outras acidemias orgânicas, e seu desenvolvimento é insidioso, sem episódios de descompensação metabólica ${ }^{36}$.

O L-2-HG é formado a partir do 2-KG através da reação catalisada pela enzima L-malato desidrogenase (L-malDH), que emprega NADPH como doador de hidretos (Figura 2). Essa reação é hipoteticamente chamada de "indesejada", pois normalmente a L-malDH catalisa a conversão de L-malato em oxaloacetato no ciclo TCA. A L-2-HGDH é considerada por alguns 


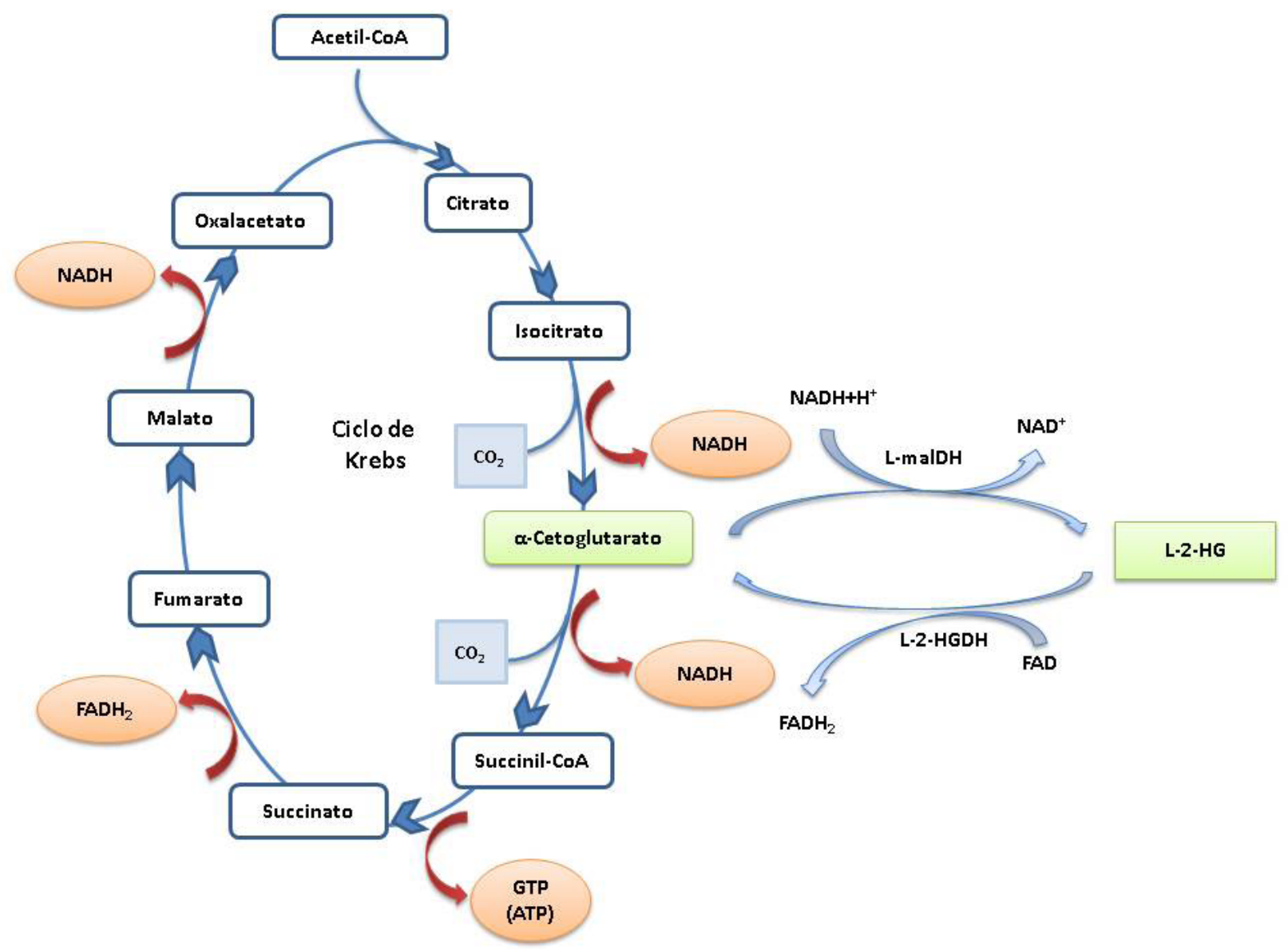

Figura 2: O ácido L-2-hidroxiglutárico (L-2-HG) é formado através da enzima L-malato desidrogenase (L-malDH) a partir de a-cetoglutarato (2-KG) e NADH. A enzima L-2-hidroxiglutarato desidrogenase (L-2-HGDH) catalisa a interconversão de L-2-HG a 2-KG utilizando FAD como cofator.

autores como enzima de reparo, pois sugere-se que com o objetivo de proteger contra potenciais efeitos tóxicos e prevenir a perda de carbonos no ciclo TCA, o L-2-HG é rapidamente reconvertido em 2-KG utilizando FAD como cofator, reparando assim a reação "indesejada" da L-malDH ${ }^{37}$.

\section{Toxicidade do ácido L-2-hidroxiglutárico}

Da Silva et al. (2003) avaliaram o efeito do L-2-HG na atividade da CK no cerebelo, córtex cerebral, músculo cardíaco e esquelético em homogeneizados de ratos Wistar, além da atividade da mi-CK e ci-CK em frações do cerebelo. O L-2-HG inibiu significativamente a CK e mi-CK no cerebelo, e este efeito inibitório, medido através de estudos cinéticos, ocorre de forma não competitiva em relação à fosfocreatina. Esses achados sugerem que a inibição seletiva da atividade dessa enzima pelo L-2-HG poderia estar relacionada à neurodegeneração cerebelar encontrada nos pacientes ${ }^{38}$.
Corroborando esses achados, outro estudo em modelo animal, que utilizou ratos knockout (I2hgdh ${ }^{-1}$ ) para a enzima L-2-HGDH, mostrou que esses ratos acumularam L-2-GH principalmente no cérebro e testículos, alcançando uma concentração de aproximadamente $3,5 \mu \mathrm{mol} / \mathrm{g}$ e valores menores em outros tecidos $(<100 \mu \mathrm{M})$. Em ratos normais, os níveis de L-2-GH foram indetectáveis. Os níveis aumentados de L-2-HG foram capazes de modificar aminoácidos no cérebro, mostrando um aumento marcado da concentração de lisina e arginina, depleção de sacaropina e diminuição de glutamina em ratos $12 \mathrm{hgdh}^{-/}$. Ainda, a constatação de que a acumulação de lisina cerebral acompanhou a supressão de sacaropina sugere que a degradação de lisina poderia prosseguir no cérebro via sacaropina e que esta via foi inibida pelo L-2-HG. Além disso, foi encontrada atividade cerebral significante, embora pequena, da enzima bifuncional lisina-alfa-cetoglutarato-redutase/sacaropina desidrogenase. Embora o efeito da acumulação 
tóxica de L-2-HG no metabolismo seja interessante do ponto de vista bioquímico, ele provavelmente não contribui para sintomas neurológicos, uma vez que defeitos no metabolismo da lisina devido à deficiência de lisina-alfa-cetoglutarato redutase não parecem ser a causa de sintomas patológicos ${ }^{39}$.

No que tange a patogenia, análises cerebrais nos ratos $\mathrm{I} 2 \mathrm{hgdh}^{-/-}$mostraram extensiva aparência espongiótica, principalmente no putâmen caudado, gânglios basais e tronco cerebral. As lesões vacuolares afetaram majoritariamente bainha de mielina e oligodendrócitos. Com relação aos testes neurocomportamentais, os ratos $12 \mathrm{hgdh}^{-1-}$ apresentaram déficit da capacidade de aprendizagem ${ }^{34}$.

Estudos do efeito histopatológico do ácido L-2-HG foram realizados através da administração aguda intracerebral in vivo de $2,5 \mu \mathrm{mol}$ do metabólito em estriado e cerebelo de ratos jovens. O ácido L-2-HG causou vacuolização intensa, infiltrado linfocítico e macrofágico, necrose, astrogliose e perda neuronal no estriado. No cerebelo foram encontradas vacuolização e infiltração linfocítica e macrofágica. Esses achados, em que o L-2-HG provoca dano in vivo cerebelar e estriatal, podem estar relacionados à indução de estresse oxidativo ${ }^{40}$.

Relatos de casos na literatura mostraram os efeitos tóxicos do L-2-HG no cérebro. Exame de ressonância magnética por imagem (RMI) confirmou leucoencefalopatia severa em paciente envolvendo, principalmente, massa branca subcortical ${ }^{41}$, hiperintensidade simétrica no núcleo dentato, globo pálido, putâmen, núcleo caudado e hiperintensidade de massa branca subcortical particularmente no lobo frontal e temporal, além de múltiplos cistos na área periventricular occiptal ${ }^{42}$.

Além de achados neuropatológicos, Isikay et al. ${ }^{42}$ relataram o caso de uma paciente de 16 anos, portadora de L-2-HGA com cardiomiopatia dilatada, caracterizada por dilatação da câmara esquerda ventricular e disfunção sistólica, que comumente resulta em insuficiência cardíaca congestiva. No entanto, essa característica pode ser secundária à acidemia ou uma coincidência. Portanto, os autores sugeriram que pacientes com L-2-HGA sejam monitorados para algum sinal de cardiomiopatia dilatada visando investigar se há correlação entre as doenças.

\section{Ácido L-2-hidroxiglutárico e câncer}

Tumores cerebrais têm sido frequentemente observados em pacientes com L-2-HGA, e esta possível associação entre tumores cerebrais malignos e a doença é o que a diferencia de outras acidúrias orgânicas ${ }^{43}$. De acordo com relatos de casos da literatura, um dos pacientes, de 20 anos, portador da doença, com níveis de $1418 \mathrm{mmol} / \mathrm{mol}$ de L-2-HG urinário (controles < 25), desenvolveu um tumor ósseo envolvendo a região frontal direita da calvária. Foi realizada análise no gene da $\mathrm{L} 2 \mathrm{HGDH}$, que revelou mutação homozigota no éxon $2(\mathrm{~A} 320 \mathrm{G})^{44}$. Outros dois pacientes, do total de um grupo de 40 , desenvolveram meduloblastoma e glioblastoma multiforme durante o curso da doença. O primeiro paciente L-2-HGA, de 3 anos, foi diagnosticado com meduloblastoma. O diagnóstico molecular realizado mostrou mutação homozigota no éxon 3 da L2HGDH na posição 292 $($ c.292C $\rightarrow T$ ), que altera o aminoácido histidina na posição 98 para tirosina (p.H98Y). O segundo paciente L-2-HGA, de 11 anos, foi diagnosticado com glioblastoma multiforme, e a análise molecular detectou mutação no éxon 7 da L2HGDH $($ c.887T $\rightarrow$ A), que altera a valina para glutamato na posição 296 $(\text { p.V296E })^{45}$. Ainda, corroborando esses achados, outro relato de caso na literatura demonstrou que, de um grupo de 11 pacientes com L-2-HGA, três pacientes desenvolveram neoplasma cerebral durante o curso da doença ${ }^{36}$, e um paciente de 29 anos foi diagnosticado com gliomatose cerebral. Além disso, não foi encontrada mutação em IDH1 e/ou IDH2 ${ }^{46}$.

Outro caso interessante na literatura é de um paciente L-2-HGA, de 22 anos, diagnosticado com astrocitoma anaplástico (OMS grau III) na região temporal direita tratado com radioterapia. No entanto, 4 anos após a terapia, exames de imagens de ressonância magnética mostraram anormalidades no lobo frontal esquerdo, sem recorrência de tumor primário ou sinais que sugerissem propagação transcalosa. Achados histopatológicos indicaram, novamente, astrocitoma anaplástico (OMS grau III). Esse foi o primeiro relato de caso de sucessivos gliomas distintos em paciente com L-2-HGA ${ }^{47}$.

Embora haja muitos relatos de casos na literatura acerca do desenvolvimento de tumores cerebrais malignos em pacientes com L-2-HGA, a relação entre esses tumores e a acidúria ainda é obscura. No entanto, algumas possibilidades são especuladas: a) quantidades similares desse metabólito estão presentes em pacientes com e sem tumor maligno cerebral, então poderia ser descartada a hipótese do envolvimento L-2-HG na oncogênese, mas, por outro lado, a situação do tecido cerebral não é refletida pela concentração do metabólito nos fluidos corporais; b) durante o processo de desmielinização e remielinização poderia haver a indução da tumorigênese devido à superexpressão de fatores de crescimento mitogênicos, mas há uma variedade de tipos tumorais e a ausência de tumores cerebrais malignos em outras doenças que remodelam a substância branca; c) mutações no gene da L2HGDH 
poderiam ter efeito oncogênico, pois com a atividade prejudicada da enzima mitocondrial ocorre acúmulo do metabólito L-2-HG no sistema nervoso central (SNC), e esse acúmulo induz estresse oxidativo, inibe a creatina cinase no cerebelo e interfere na transmissão glutamatérgica ${ }^{45}$.

\section{Ácido L-2-hidroxiglutárico e o envolvimento no estresse oxidativo}

A administração aguda intracerebral in vivo de 2,5 $\mathrm{umol}$ de L-2-HG em ratos jovens mostrou que o ácido acumulado provoca estresse oxidativo. Esse ácido acumulado diminuiu significativamente as defesas antioxidantes enzimáticas, como a atividade de GPx e GR, não enzimáticas como GSH, e também aumentou os níveis de GSSH no estriado e cerebelo. Além disso, houve aumento de ERO, que foi determinado por oxidação de DCFH e produção de $\mathrm{H}_{2} \mathrm{O}_{2}$, além do aumento de MDA, que reflete dano lipídico. Cabe salientar que essas alterações na homeostase redox causadas pelo L-2-HGA foram seletivas, tendo em vista que foi utilizado um análogo estruturalmente similar (ácido L-2-hidroxi-3metil-valérico), o qual não alterou nenhum desses parâmetros ${ }^{40}$. Além disso, esses resultados em modelo animal estão de acordo com um estudo realizado em pacientes portadores de L-2-HGA, no qual foram medidos alguns parâmetros em plasma. As análises mostraram aumento significativo dos níveis plasmáticos de MDA, diminuição significativa das atividades de SOD e GPx, e redução da concentração de GSH na comparação com os controles. Esses resultados evidenciam que o L-2-HG acumulado no plasma dos pacientes pode causar toxicidade, levando a um desequilíbrio redox e, consequentemente, ao estresse oxidativo ${ }^{48}$.

\section{Abordagem terapêutica}

A suplementação com FAD e seu precursor, a riboflavina, foi usada em alguns pacientes com o objetivo de fomentar a atividade residual da enzima L-2-HGD, uma vez que a enzima é dependente de FAD. Além disso, esperou-se promover o aumento da oxidação de L-2-HGA e talvez, assim, reduzir sua toxicidade. No entanto, a eficiência do uso terapêutico de cofatores depende de diversos fatores, como a atividade residual da enzima e se a mutação tem impacto significativo na interação enzima-cofator. De acordo com relato na literatura, um paciente com L-2-HGA, de 16 anos, recebeu inicialmente $100 \mathrm{mg} / \mathrm{dia}$ de riboflavina, e foi observada melhora parcial em seu desempenho motor e cognitivo, além da diminuição significativa da excreção urinária do L-2-HG. Entretanto, houve uma interrupção do tratamento e os distúrbios ficaram acentuados, com aumento da excreção do ácido. Após o reestabelecimento do tratamento com riboflavina, o paciente voltou ao status clínico anterior. A melhora aumentou minimamente com a dose de $200 \mathrm{mg} / \mathrm{dia}$, mas não se alterou na dose de $300 \mathrm{mg} / \mathrm{dia}^{49}$. Em outro relato de caso da literatura, no qual o paciente foi submetido a terapia com riboflavina (200 mg/dia), além de carnitina (1 g/dia), ele apresentou sintomas estáveis durante o período de 1 ano pelo qual foi acompanhado na clínica ${ }^{50}$. Apesar disso, o tratamento farmacológico com vitaminas às vezes tem resultados ótimos, outras vezes, não. Sabe-se que pacientes com certas doenças têm respostas variáveis a determinado tratamento. A exemplo disso, Jovic et al. ${ }^{30}$ mostraram um caso de paciente no qual a terapia com riboflavina $(200 \mathrm{mg} / \mathrm{dia})$ por 4 meses não levou a uma diferença significativa nos parâmetros clínicos e/ou bioquímicos.

\section{ACIDÚRIA D,L-2-HIDROXIGLUTÁRICA}

AD,L-2-HGA é uma rara doença neurometabólica bioquimicamente caracterizada por excreção aumentada de L-2-HG e D-2-HG, com predomínio de D-2-HG e alfa-cetoglutarato, bem como uma diminuição de citrato urinário. Ao contrário dos casos de D-2-HGA e L-2-HGA, esses pacientes apresentam ambos os enantiômeros acumulados do ácido 2-hidroxiglutárico. $\mathrm{Na}$ D,L-2-HGA, a mutação ocorre no gene SLC25A1, que codifica o carreador de citrato mitocondrial $(\mathrm{CIC})$. O CIC medeia o efluxo de intermediários do ciclo TCA, citrato e isocitrato em troca do malato citosólico. Dessa forma, a depleção de citrato citosólico, bem como a acumulação de citrato dentro da mitocôndria, tem sido considerada importante no desenvolvimento da patofisiologia dessa acidúria ${ }^{51}$.

Como sintomas clínicos, esses pacientes apresentam hipotonia muscular, disfunção severa de neurodesenvolvimento e convulsões intratáveis associadas à dificuldade respiratória ${ }^{51}$.

\section{Abordagem terapêutica}

De acordo com um relato de caso na literatura referente à abordagem terapêutica, avaliou-se o uso de malato e citrato como perspectiva de tratamento. Nesse estudo, um paciente de cinco meses de idade, diagnosticado com acidemia D,L-2-hidroxiglutárica foi tratado com malato ou citrato. Durante o tratamento com malato, houve aumento da concentração urinária de malato, mas não foi observada melhora bioquímica ou clínica. Entretanto, quando o paciente foi tratado com citrato, houve um aumento da excreção urinária dos intermediários do ciclo do ácido cítrico, malato e succinato, além de um leve aumento na concentração 
do citrato urinário. Dessa forma, a excreção dos ácidos D,L-2-HG diminuiu durante o tratamento. A resposta clínica foi a estabilização na frequência e na gravidade das convulsões ${ }^{52}$.

\section{DIAGNÓSTICO}

A suspeita do diagnóstico ocorre através de avaliação clínica dos pacientes com atraso de desenvolvimento e outras disfunções neurológicas de etiologia desconhecida. No entanto, devido às características clínicas geralmente homogêneas, a suspeita de diagnóstico pode ocorrer de forma tardia, pois a doença tem um curso lento e progressivo, sem sinais clínicos peculiares ${ }^{36}$. Aliado aos sintomas clínicos, a confirmação da doença é realizada através de CG/MS para detecção de altos níveis do ácido orgânico na urina. No entanto, para classificar como D ou L-2-HG é necessário fazer a diferenciação quiral ${ }^{53}$. Além disso, a análise molecular é uma importante ferramenta para confirmação do diagnóstico através da detecção da mutação e bastante útil para aconselhamento genético ${ }^{32}$.

\section{CONSIDERAÇÕES FINAIS}

As acidúrias neurometabólicas D-2-HGA, L-2-HGA ou D,L-2-HGA são distintas entre si, e os mecanismos que envolvem a patogenicidade ainda permanecem obscuros. Alguns estudos demostraram o efeito tóxico do acúmulo dos ácidos correspondentes tanto em modelos animais quanto em humanos. Ainda, alguns autores mostraram esses ácidos como agentes causadores de estresse oxidativo, o que acarreta alteração da homeostase redox e leva a uma série de consequências em nível cerebral. No entanto, muito ainda precisa ser elucidado. Além disso, os ácidos D-2-HG e L-2-HG foram detectados em altos níveis em tumores cerebrais. Mutações encontradas nas enzimas IDH1 e IDH2 levaram ao questionamento do papel desses ácidos, levantando a hipótese de que eles agem como possíveis oncometabólitos. Entretanto, a relação entre essas acidúrias e o câncer ainda é desconhecido e são necessários muitos estudos para melhor entender essa questão.

\section{REFERÊNCIAS}

1. Vaidyanathan K, Narayanan MP, Vasudevan DM. Organic acidurias: an updated review. Indian J Clin Biochem. 2011;26(4):319-25. http:// dx.doi.org/10.1007/s12291-011-0134-2. PMid:23024466.

2. Scriver CR, Beaudet AL, Sly WS, Valle D. The metabolic and molecular bases of inherited disease. 8th ed. New York: McGraw-Hill; 2001.

3. Steenweg ME, Salomons GS, Yapici Z, Uziel G, Scalais E, Zafeiriou DI, et al. L-2-Hydroxyglutaric aciduria: pattern of MR imaging abnormalities in 56 patients. Radiology. 2009;251(3):85665. http://dx.doi.org/10.1148/ radiol.2513080647. PMid:19474378.

4. Chalmers RA, Lawson AM, Watts RW, Tavill AS, Kamerling JP, Hey E, et al. D-2-hydroxyglutaric aciduria: case report and biochemical studies. J Inherit Metab Dis. 1980;3(1):11-5. http://dx.doi.org/10.1007/BF02312516. PMid:6774165.

5. Duran M, Kamerling JP, Bakker HD, van Gennip AH, Wadman SK. L-2-Hydroxyglutaric aciduria: an inborn error of metabolism? J Inherit Metab Dis. 1980;3(1):109-12. http:// dx.doi.org/10.1007/BF02312543. PMid:6787330.
6. Muntau AC, Röschinger $W$, Merkenschlager $A$, van der Knaap MS, Jakobs C, Duran $\mathrm{M}$, et al. Combined D-2- and L-2-hydroxyglutaric aciduria with neonatal onset encephalopathy: a third biochemical variant of 2-hydroxyglutaric aciduria? Neuropediatrics. 2000;31(3):137-40. http://dx.doi. org/10.1055/s-2000-7497. PMid:10963100.

7. Kranendijk M, Struys EA, Salomons GS, Van der Knaap MS, Jakobs C. Progress in understanding 2-hydroxyglutaric acidurias. J Inherit Metab Dis. 2012;35(4):571-87. http:// dx.doi.org/10.1007/s10545-012-94625. PMid:22391998.

8. Struys EA. D-2-Hydroxyglutaric aciduria: unravelling the biochemical pathway and the genetic defect. $J$ Inherit Metab Dis. 2006;29(1):21-9. http://dx.doi.org/10.1007/s10545-0060317-9. PMid:16601864.

9. Balaji $P$, Viswanathan $V$, Chellathurai A, Panigrahi D. An interesting case of metabolic dystonia: L-2hydroxyglutaric aciduria. Ann Indian Acad Neurol. 2014;17(1):97-9. http://dx.doi.org/10.4103/09722327.128565. PMid:24753671.
10. Wajner M, Goodman SI. Disruption of mitochondrial homeostasis in organic acidurias: insights from human and animal studies. J Bioenerg Biomembr. 2011;43(1):31-8. http://dx.doi. org/10.1007/s10863-011-9324-0. PMid:21249436.

11. Gibson KM, ten Brink HJ, Schor DS, Kok RM, Bootsma AH, Hoffmann GF, et al. Stable-isotope dilution analysis of D- and L-2-hydroxyglutaric acid: application to the detection and prenatal diagnosis of $\mathrm{D}$-and L-2hydroxyglutaric acidemias. Pediatr Res. 1993;34(3):277-80. http://dx.doi. org/10.1203/00006450-19930900000007 . PMid:8134166.

12. Kranendijk M, Struys EA, Gibson $\mathrm{KM}$, Wickenhagen WV, Abdenur JE, Buechner J, et al. Evidence for genetic heterogeneity in D-2-hydroxyglutaric aciduria. Hum Mutat. 2010;31(3):27983. http://dx.doi.org/10.1002/ humu.21186. PMid:20020533.

13. Kranendijk M, Struys EA, van Schaftingen E, Gibson KM, Kanhai WA, van der Knaap MS, et al. IDH2 mutations in patients with D-2hydroxyglutaric aciduria. Science. 2010;330(6002):336. http://dx.doi. org/10.1126/science.1192632. PMid:20847235. 
14. Bhagwat N, Levine RL. Metabolic syndromes and malignant transformation: where the twain shall meet. Sci Transl Med. 2010;2(54):54ps50. http://dx.doi. org/10.1126/scitranslmed.3001669. PMid:20962328.

15. Kranendijk M, Salomons GS, Gibson KM, Van Schaftingen E, Jakobs C, Struys EA. A lymphoblast model for IDH2 gain-of-function activity in d-2-hydroxyglutaric aciduria type II: novel avenues for biochemical and therapeutic studies. Biochim Biophys Acta. 2011;1812:1380-4.

16. Kölker S, Pawlak V, Ahlemeyer B, Okun JG, Hörster F, Mayatepek $\mathrm{E}$, et al. NMDA receptor activation and respiratory chain complex $V$ inhibition contribute to neurodegeneration in d-2-hydroxyglutaric aciduria. Eur J Neurosci. 2002;16(1):21-8 http://dx.doi.org/10.1046/j.14609568.2002.02055.x. PMid:12153528.

17. Silva CG, Bueno AR, Schuck PF, Leipnitz G, Ribeiro CA, Wannmacher CM, et al. D-2hydroxyglutaric acid inhibits creatine kinase activity from cardiac and skeletal muscle of young rats. Eur J Clin Invest. 2003;33(10):840-7. http://dx.doi.org/10.1046/j.13652362.2003.01237.x. PMid:14511354.

18. Silva CG, Bueno AR, Schuck PF, Leipnitz G, Ribeiro CA, Rosa $\mathrm{RB}$, et al. Inhibition of creatine kinase activity from rat cerebral cortex by D-2-hydroxyglutaric acid in vitro. Neurochem Int. 2004;44(1):45-52. http://dx.doi.org/10.1016/S01970186(03)00098-6. PMid:12963087.

19. Latini A, Silva CG, Ferreira GC, Schuck PF, Scussiato K, Sarkis $\mathrm{JJ}$, et al. Mitochondrial energy metabolism is markedly impaired by D-2-hydroxyglutaric acid in rat tissues. Mol Genet Metab. 2005;86(12):188-99. http://dx.doi.org/10.1016/j. ymgme.2005.05.002. PMid:15963747.

20. Rosa MS, Seminotti B, Amaral $\mathrm{AU}$, Parmeggiani B, Oliveira $\mathrm{FH}$, Leipnitz G, et al. Disruption of redox homeostasis and histopathological alterations caused by in vivo intrastriatal administration of D-2hydroxyglutaric acid to young rats. Neuroscience. 2014;277:28193. http://dx.doi.org/10.1016/j. neuroscience.2014.07.011. PMid:25043325.

21. Parsons DW, Jones $S$, Zhang $X$, Lin JC, Leary RJ, Angenendt P, et al. An integrated genomic analysis of human glioblastoma multiforme. Science. 2008;321(5897):1807-12. http:// dx.doi.org/10.1126/science.1164382. PMid:18772396.

22. Rakheja D, Mitui M, Boriack $\mathrm{RL}$, DeBerardinis RJ. Isocitrate dehydrogenase $1 / 2$ mutational analyses and 2-hydroxyglutarate measurements in Wilms tumors. Pediatr Blood Cancer. 2011;56(3):37983. http://dx.doi.org/10.1002/ pbc.22697. PMid:21225914.

23. Ward PS, Patel J, Wise DR, AbdelWahab O, Bennett BD, Coller $\mathrm{HA}$, et al. The common feature of leukemia-associated IDH1 and IDH2 mutations is a neomorphic enzyme activity converting alpha-ketoglutarate to 2-hydroxyglutarate. Cancer Cell. 2010;17(3):225-34. http://dx.doi. org/10.1016/j.ccr.2010.01.020. PMid:20171147.

24. Gross S, Cairns RA, Minden MD, Driggers EM, Bittinger MA, Jang HG, et al. Cancer-associated metabolite 2-hydroxyglutarate accumulates in acute myelogenous leukemia with isocitrate dehydrogenase 1 and 2 mutations. $J$ Exp Med. 2010;207(2):339-44. http:// dx.doi.org/10.1084/jem.20092506. PMid:20142433.

25. Rakheja D, Boriack RL, Mitui M, Khokhar S, Holt SA, Kapur P. Papillary thyroid carcinoma shows elevated levels of 2-hydroxyglutarate. Tumour Biol. 2011;32(2):325-33. http://dx.doi. org/10.1007/s13277-010-0125-6. PMid:21080253.

26. Cohen AL, Holmen SL, Colman $\mathrm{H}$. IDH1 and IDH2 mutations in gliomas. Curr Neurol Neurosci Rep. 2013;13(5):345. http://dx.doi. org/10.1007/s11910-013-0345-4. PMid:23532369.

27. Rakheja D, Fuda F, Vandergriff T, Boriack R, Medeiros BC, Frankel $A E$, et al. Increased plasma d-2hydroxyglutarate in isocitrate dehydrogenase 2-mutated blastic plasmacytoid dendritic cell neoplasm. Hum Pathol. 2015;46(2):322-6. http://dx.doi. org/10.1016/j. humpath.2014.10.013. PMid:25481493.

28. Latini A, Scussiato $K$, Rosa RB, Llesuy S, Belló-Klein A, DutraFilho CS, et al. D-2-hydroxyglutaric acid induces oxidative stress in cerebral cortex of young rats. Eur J Neurosci. 2003;17(10):2017-22. http://dx.doi.org/10.1046/j.14609568.2003.02639.x. PMid:12786967.

29. Matsunaga $H$, Futakuchi-Tsuchida $A$, Takahashi M, Ishikawa T, Tsuji M, Ando $\mathrm{O}$. IDH1 and IDH2 have critical roles in 2-hydroxyglutarate production in D-2-hydroxyglutarate dehydrogenase depleted cells. Biochem Biophys Res Commun. 2012;423(3):553-6. http:// dx.doi.org/10.1016/j.bbrc.2012.06.002. PMid:22683334.

30. Jović NJ, Kosać A, Koprivsek K. L-2-Hydroxyglutaric aciduria: a case report. Srp Arh Celok Lek. 2014;142(56):337-41. http://dx.doi.org/10.2298/ SARH1406337J. PMid:25033591.

31. Faiyaz-UI-Haque M, Al-Sayed MD, Faqeih E, Jamil M, Saeed A, Amoudi $M S$, et al. Clinical, neuroimaging, and genetic features of L-2-hydroxyglutaric aciduria in Arab kindreds. Ann Saudi Med. 2014;34(2):107-14. PMid:24894778.

32. Rzem R, Veiga-da-Cunha M, Noël G, Goffette S, Nassogne MC, Tabarki $B$, et al. A gene encoding a putative FAD-dependent L-2-hydroxyglutarate dehydrogenase is mutated in L-2hydroxyglutaric aciduria. Proc Natl Acad Sci USA. 2004;101(48):1684954. http://dx.doi.org/10.1073/ pnas.0404840101. PMid:15548604.

33. Steenweg ME, Jakobs C, Errami A, van Dooren SJ, Adeva Bartolomé MT, Aerssens P, et al. An overview of L-2-hydroxyglutarate dehydrogenase gene (L2HGDH) variants: a genotype-phenotype study. Hum Mutat. 2010;31(4):380-90. http:// dx.doi.org/10.1002/humu.21197. PMid:20052767.

34. Marcel C, Mallaret M, Lagha-Boukbiza O, Kremer S, Echaniz-Laguna A, Tranchant C. L-2-hydroxyglutaric aciduria diagnosed in a young adult with progressive cerebellar ataxia and facial dyskinesia. Rev Neurol (Paris). 2012;168(2):187-91. http://dx.doi. org/10.1016/j.neurol.2011.06.002. PMid:22030381.

35. Mazzei R, Ungaro C, Garreffa G, Conforti FL, Mollo A, Sprovieri T, et al. Clinical, genetic and magnetic resonance findings in an Italian patient affected by L-2-hydroxyglutaric aciduria. Neurol Sci. 2011;32(1):95-9. http://dx.doi.org/10.1007/s10072-0100416-0. PMid:20859647.

36. Patay Z, Mills JC, Löbel U, Lambert A, Sablauer A, Ellison DW. Cerebral neoplasms in L-2-hydroxyglutaric 
aciduria: 3 new cases and metaanalysis of literature data. AJNR Am J Neuroradiol. 2012;33(5):940-3. http://dx.doi.org/10.3174/ajnr.A2869. PMid:22241392.

37. Van Schaftingen E, Rzem R, Veigada-Cunha ML. -2-Hydroxyglutaric aciduria, a disorder of metabolite repair. J Inherit Metab Dis. 2009;32(2):135-42. http://dx.doi. org/10.1007/s10545-008-1042-3. PMid:19020988.

38. Silva CG, Bueno AR, Schuck PF, Leipnitz G, Ribeiro CA, Wannmacher $\mathrm{CM}$, et al. L-2-hydroxyglutaric acid inhibits mitochondrial creatine kinase activity from cerebellum of developing rats. Int J Dev Neurosci. 2003;21(4):217-24. http://dx.doi. org/10.1016/S0736-5748(03)00035-2. PMid:12781789.

39. Rzem R, Achouri Y, Marbaix E, Schakman O, Wiame E, Marie $\mathrm{S}$, et al. A mouse model of L-2hydroxyglutaric aciduria, a disorder of metabolite repair. PLoS One. 2015;10(3):e0119540. http://dx.doi. org/10.1371/journal.pone.0119540. PMid:25763823.

40. Rosa MS, Ribeiro CAJ, Seminotti B, Ribeiro RT, Amaral AU, Coelho $\mathrm{DM}$, et al. In vivo intracerebral administration of L-2-hydroxyglutaric acid provokes oxidative stress and histopathological alterations in striatum and cerebellum of adolescent rats. Free Radic Biol Med. 2015;83:201-13. http://dx.doi.org/10.1016/j. freeradbiomed.2015.02.008. PMid:25701435.

41. Weimar C, Schlamann M, Krägeloh-Mann I, Schöls L. L-2 hydroxyglutaric aciduria as a rare cause of leukencephalopathy in adults. Clin Neurol Neurosurg. 2013;115(6):765-6. http://dx.doi. org/10.1016/j.clineuro.2012.06.040. PMid:22840416.
42. Işıkay S, Ceylaner S, Karacan M. A child with L-2 hydroxyglutaric aciduria presenting with dilated cardiomyopathy: coincidence or a new syndrome? Anadolu Kardiyol Derg. 2014;14(1):92-3. PMid:24382499.

43. Vilarinho L, Cardoso ML, Gaspar P, Barbot C, Azevedo L, Diogo L, et al. Novel L2HGDH mutations in 21 patients with L-2-hydroxyglutaric aciduria of Portuguese origin. Hum Mutat. 2005;26(4):395-6. http:// dx.doi.org/10.1002/humu.9373. PMid:16134148.

44. Larnaout A, Amouri R, Neji S, Zouari M, Kaabachi N, Hentati F. Osteoma of the calvaria in L-2-hydroxyglutaric aciduria. J Inherit Metab Dis. 2007;30(6):980. http://dx.doi. org/10.1007/s10545-007-0576-0. PMid:17917788.

45. Haliloglu G, Jobard F, Oguz KK, Anlar B, Akalan N, Coskun T, et al. L-2-hydroxyglutaric aciduria and brain tumors in children with mutations in the L2HGDH gene: neuroimaging findings. Neuropediatrics. 2008;39(2):119-22. http://dx.doi. org/10.1055/s-2008-1081217. PMid:18671189.

46. London F, Jeanjean A. Gliomatosis cerebri in L-2-hydroxyglutaric aciduria. Acta Neurol Belg. 2015;115(4):749-51. http://dx.doi.org/10.1007/s13760-0150489-x. PMid:25997888.

47. Patay Z, Orr BA, Shulkin BL, Hwang SN, Ying Y, Broniscer A, et al. Successive distinct high-grade gliomas in L-2-hydroxyglutaric aciduria. J Inherit Metab Dis. 2015;38(2):273-7. http://dx.doi. org/10.1007/s10545-014-9782-8. PMid:25338511.

48. Jellouli NK, Hadj Salem I, Ellouz E, Kamoun Z, kamoun F, tili A, et al. Founder effect confirmation of c. $241 \mathrm{~A}>\mathrm{G}$ mutation in the $\mathrm{L} 2 \mathrm{HGDH}$ gene and characterization of oxidative stress parameters in six Tunisian families with L-2hydroxyglutaric aciduria. J Hum Genet. 2014;59(4):216-22. http:// dx.doi.org/10.1038/jhg.2014.4 . PMid:24573090.

49. Yilmaz K. Riboflavin treatment in a case with l-2-hydroxyglutaric aciduria. Eur J Paediatr Neurol. 2009;13(1):5760. http://dx.doi.org/10.1016/j. ejpn.2008.01.003. PMid:18343698.

50. Işıkay S. Cerebral multicystic lesions in a child with L-2 hydroxyglutaric aciduria: a rare disease and a rare association. Pediatr Neurol. 2014;50(2):1978. http://dx.doi.org/10.1016/j. pediatrneurol.2013.05.017. PMid:24314673.

51. Nota B, Struys EA, Pop A, Jansen EE, Ojeda MRF, Kanhai WA, et al. Deficiency in SLC25A1, encoding the mitochondrial citrate carrier, causes combined D-2- and L-2hydroxyglutaric aciduria. Am J Hum Genet. 2013;92(4):627-31. http:// dx.doi.org/10.1016/j.ajhg.2013.03.009. PMid:23561848.

52. Mühlhausen C, Salomons GS, Lukacs Z, Struys EA, van der Knaap MS, Ullich K, et al. Combined D2-/L2hydroxyglutaric aciduria (SLC25A1 deficiency): clinical course and effects of citrate treatment. J Inherit Metab Dis. 2014;37(5):775-81. http://dx.doi. org/10.1007/s10545-014-9702-y. PMid:24687295.

53. Struys EA, Jansen EE, Verhoeven NM, Jakobs C. Measurement of urinary D- and L-2-hydroxyglutarate enantiomers by stable-isotopedilution liquid chromatographytandem mass spectrometry after derivatization with diacetyl-Ltartaric anhydride. Clin Chem. 2004;50(8):1391-5. http://dx.doi. org/10.1373/clinchem.2004.033399. PMid:15166110.

Recebido: Dez 21, 2015 Aceito: Abr 16, 2016 